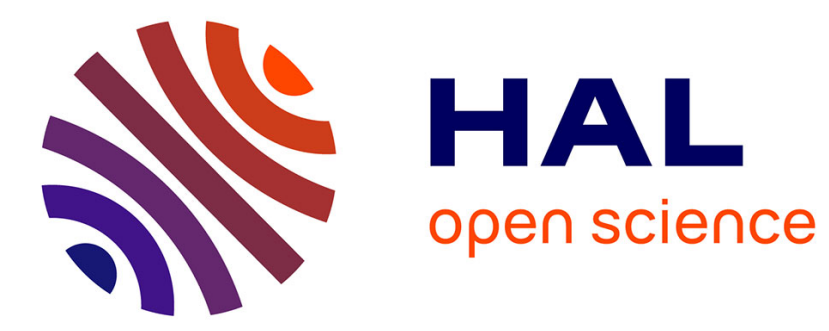

\title{
Propriétés et applications des alliages intermétalliques B2-FeAl
}

\author{
F. Moret, R. Baccino, P. Martel, L. Guetaz
}

\section{To cite this version:}

F. Moret, R. Baccino, P. Martel, L. Guetaz. Propriétés et applications des alliages intermétalliques B2FeAl. Journal de Physique IV Proceedings, 1996, 06 (C2), pp.C2-281-C2-289. 10.1051/jp4:1996240 . jpa-00254218

\section{HAL Id: jpa-00254218 https://hal.science/jpa-00254218}

Submitted on 1 Jan 1996

HAL is a multi-disciplinary open access archive for the deposit and dissemination of scientific research documents, whether they are published or not. The documents may come from teaching and research institutions in France or abroad, or from public or private research centers.
L'archive ouverte pluridisciplinaire HAL, est destinée au dépôt et à la diffusion de documents scientifiques de niveau recherche, publiés ou non, émanant des établissements d'enseignement et de recherche français ou étrangers, des laboratoires publics ou privés. 


\title{
Propriétés et applications des alliages intermétalliques B2-FeAl
}

\author{
F. Moret, R. Baccino, P. Martel et L. Guetaz \\ CEA/CEREM, DEM/Section de Génie des Matériaux CENG, 38054 Grenoble cedex 9, France
}

\begin{abstract}
Ordered FeAl intermetallic alloys are attractive materials for medium and high temperature industrial applications but their use has been restricted until now by their room temperature brittleness and their poor creep resistance. Powder metallurgy $(\mathrm{P} / \mathrm{M})$ techniques such as gas atomization and mechanical milling have been used to develop FeAl alloys with enhanced ductility and strength at both low and high temperatures. The improvement method combines ductilization by grain boundary strengthening, grain size reduction and oxide dispersion strengthening. These materials named FeAl40 Grade 3 have been characterized and tested in the form of extruded bars. Microstructure and texture of as-extruded and heat treated samples have been studied by TEM and X-ray diffraction. Grains are $0,5 \mu \mathrm{m}$ in size, resistant to recrystallization up to $1100^{\circ} \mathrm{C}$ and exhibit a strong $\langle 110\rangle$ wire texture parallel to the extrusion axis. The $\mathrm{Y}_{2} \mathrm{O}_{3}$ dispersoüds $(20-30 \mathrm{~nm}$ in size) are cylindrical in shape and partially coherent with the matrix. The yield strength and the elongation of such alloys can reach $900 \mathrm{Mpa}$ and $6,4 \%$ in air. Physical and mechanical properties of these materials are compared to some conventional engineering alloys in order to discuss the conceivable applications in aeronautical and automotive industries. Due to the high specific stiffness and strength of FeAl40 Grade 3 alloys, promising applications are the substitutions of steels and superalloys for the fabrication of moving parts in thermal and aeronautical engines, and especially parts submitted to critical vibrating modes.
\end{abstract}

\section{INTRODUCTION}

Les aluminiures de fer basés sur le composé ordonné équi-atomique $\mathrm{FeAl}$ (structure cubique centrée de type B2) possèdent une excellente résistance en environnement oxydant, sulfurant ou carburant jusqu'à $1000^{\circ} \mathrm{C}$ et une bonne résistance à l'érosion. Ces propriétés associées à leur densité relativement faible $(<6$ g.cm ${ }^{-3}$ ), et au coût réduit des matériaux de base [1-2], leur procure un intérêt potentiel pour la fabrication de pièces structurales, y compris dans des environnements agressifs pour des applications telles que des échangeurs de chaleur ou encore des composants de moteurs ou de turbines. Quoique ces alliages aient été étudiés depuis de nombreuses années [3], leur utilisation a été jusqu'à présent retardée par deux inconvénients majeurs: leur fragilité à température ambiante, et leur faible résistance au fluage (commune aux structures cubiques centrées).

Depuis les années soixante, la ductilisation des alliages FeAl à température ambiante a fait l'objet de nombreuses recherches. Contrairement aux alliages stoechiométriques $\mathrm{Fe}-50 \% \mathrm{Al}$ qui sont complètement fragiles [4-6], il a été montré qu'une plasticité limitée peut être obtenue en réduisant la teneur en aluminium. Toutefois, la rupture reste intergranulaire de façon prédominante [5-10]. Les systèmes de glissements $<111>\{110\}$ de ces alliages étant connus pour être actifs à basse température [11-14], cette rupture intergranulaire n'est pas la conséquence d'un non respect du critère de Von-Misès, qui indique que cinq systèmes de glissements actifs indépendants sont nécessaires pour permettre la déformation plastique d'un matériau polycristallin. Basés sur ces observations, les premiers travaux visant à ductiliser les intermétalliques $\mathrm{FeAl}$ sous-stoechiométriques ont recherché à améliorer la résistance des joints de grains. 
Des traitements thermiques favorisant la formation de carbures ont permis d'améliorer la ductilité de ces intermétalliques en réduisant la ségrégation de carbone libre aux joints de grains. Sainfort et al. [3] ont montré qu'un tel résultat était obtenu par une addition de $\mathrm{Zr}$ jusqu'à $0,1 \%$ pds. De même que pour l'intermétallique $\mathrm{Ni}_{3} \mathrm{Al}$, un dopage au bore améliore la résistance du joint de grains, ce qui se traduit par une rupture transgranulaire par clivage [4-7,9]. Toutefois, cette concentration doit être optimisée [4-5] car, aux grandes concentrations en B, l'effet de durcissement des atomes de B interstitiels augmente la limite élastique et la rupture par clivage se produit avant que le domaine plastique ne soit atteint. L'effet du bore n'apparaît donc plus bénéfique pour la ductilité.

La ductilité à température ambiante de alliages intermétalliques FeAl sous-stoechiométriques peut aussi être améliorée par un refroidissement lent depuis des températures élevées afin de réduire la concentration en défauts ponctuels [5-6]. Il en résulte une réduction de la limite élastique qui permet d'observer une plasticité pour des contraintes inférieures à celle de la décohésion intergranulaire. Un tel comportement a également été observé pour des alliages dopés au $\mathrm{B}$, mais l'effet est moins prononcé que pour des alliages ne contenant pas de bore.

La faible résistance des joints de grains n'est pas la seule cause de fragilité à température ambiante. Des travaux récents ont révélé une augmentation significative de la ductilité quand l'atmosphère d'essai est sèche $[1,6,9-10]$. Liu et al. [9-10] ont proposé une interprétation basée sur le dégagement d'hydrogène fragilisant en fond de fissure, résultant de la formation d'alumine par la réaction de la vapeur d'eau de l'atmosphère avec les atomes d'aluminium mis à nu.

Nagpal et Baker [15] ont montré que l'allongement du Fe-45\%Al sous air est indépendant de la vitesse de déformation jusqu'à $10^{-2} \mathrm{~s}^{-1}$, mais augmente avec la vitesse de traction jusqu'à $1 \mathrm{~s}^{-1}$. Webb et Lefort [16] ont étudié les effets couplés de la vitesse de déformation et de l'environnement sur la ductilité à l'ambiante d'un alliage $\mathrm{Fe}-40 \% \mathrm{Al}$ à teneurs optimisées en $\mathrm{Zr}$ et $\mathrm{B}$. Ils ont observé que la ductilité est considérablement améliorée avec l'augmentation de la vitesse de traction quand les essais sont réalisés à l'air. Au contraire, quand les essais sont réalisés sous vide, la ductilité ne dépend pas de la vitesse de déformation. Les fractographies montrent que la fragilité provient d'une rupture par clivage qui apparaît en premier lieu dans les grains affectés en périphérie des éprouvettes. En conséquence, il a été proposé que la fragilisation par l'environnement à température ambiante des intermétalliques $\mathrm{Fe}-\mathrm{Al}$ riches en fer résulte d'une perte de résistance, dépendant du temps, des plans de clivage des grains affectés. Suivant l'hypothèse de Liu et al. citée précédemment, cet affaiblissement des plans de clivage serait dû à l'absorption d'hydrogène produit par la décomposition de la vapeur en fond de fissure. Eliminer la vapeur d'eau de l'atmosphère d'essai (respectivement augmenter la vitesse de traction) augmente la ductilité en éliminant les (respectivement en réduisant le nombre des) grains affectés par cette diffusion d'hydrogène.

\section{DEFINITION ET FABRICATION D'ALliages FeAl A DUCTILITE A L'AMBIANTE AMELIOREE}

Webb et Lefort [16] ont combiné les concepts connus sur la fragilité à température ambiante des intermétalliques $\mathrm{FeAl}$ riches en fer pour construire un modèle schématique prenant en compte les variables matériau et les paramètres expérimentaux. Ce modèle illustre les effets la dépendance en taille de grains de la limite élastique, de la résistance des joints de grains et de la résistance au clivage. Ce modèle a été utilisé pour concevoir un alliage intermétallique FeAl riche en fer présentant une ductilité maximale en température et atmosphère ambiantes, associée à une résistance mécanique et une tenue au fluage suffisantes pour justifier des applications [17]. Cette optimisation de propriétés a été obtenue en améliorant simultanément la résistance des joints de grains par des additions de $\mathrm{B}$ et de $\mathrm{Zr}$, la résistance 
au clivage en réduisant la taille de grains, et la résistance au fluage à l'aide d'une dispersion intragranulaire fine et stable.

La composition de cet alliage ( $\mathrm{Fe}-24 \% \mathrm{Al}-0,11 \% \mathrm{Zr}-15 \mathrm{ppm} \mathrm{B}$ en poids), désigné sous le nom de FeAl40 Grade 3, a été choisie pour donner le maximum de renforcement des joints de grains. La taille de grains fine a été obtenue par l'utilisation de techniques de métallurgie des poudres incluant une étape de broyage mécanique. Après son élaboration, l'alliage est atomisé sous argon. La poudre pré-alliée obtenue est broyée très énergiquement par des billes d'acier sous atmosphère contrôlée dans un broyeur à boulets. L'accumulation de défauts structuraux introduits par les chocs répétés induit la formation de grains cristallographiques très fins [18], de quelques dizaines de nanomètres. Le broyage mécanique est utilisé pour introduire simultanément dans le matériau une dispersion nanométrique d'un oxyde très stable, $\mathrm{Y}_{2} \mathrm{O}_{3}$, à partir de poudre de ce composé, en proportion de $1 \%$ poids. Cette dispersion très fine a deux effets. En premier lieu, elle limite la recristallisation au cours de l'étape de consolidation par filage à chaud qui suit le broyage. En second lieu, elle améliore considérablement la résistance au fluage et la stabilité thermique [19].

Des travaux récents ont conduit à optimiser le processus de fabrication de l'alliage FeAl40 Grade 3 [20]. Dans une première étape, les lingots d'alliage mère présentant la composition mentionnée précédemment sont coulés à partir de métaux élémentaires. Puis, ce matériau est atomisé sous argon et tamisé afin d'obtenir une poudre fine et sphérique. Le broyage à sec est réalisé sous argon dans un broyeur à boulets rotatif de capacité égale à $10 \mathrm{~kg}$ de poudre par lot, et chargé avec $100 \mathrm{~kg}$ de billes d'acier au carbone (fig. 1). La poudre d'oxyde d'yttrium est ajoutée au début du broyage. Toutes les opérations de manipulation sont réalisées dans des conditions très propres pour limiter la contamination par l'atmosphère ou par des inclusions exogènes. Les analyses chimiques avant et après broyage ont montré que cette opération introduisait une quantité d'environ $1000 \mathrm{ppm}$ en poids d'oxygène dans le matériau. Une contamination en carbone d'environ $200 \mathrm{ppm}$ en poids est introduite par l'usure des billes au cours du broyage.

La poudre broyée est mise en conteneur, dégazée et extrudée à $1100^{\circ} \mathrm{C}$ pour produire des barres d'un diamètre variant de 10 à $40 \mathrm{~mm}$ (fig. 2). A ce jour, une centaine de kilogramme de matériau a été produite suivant cette procédure.

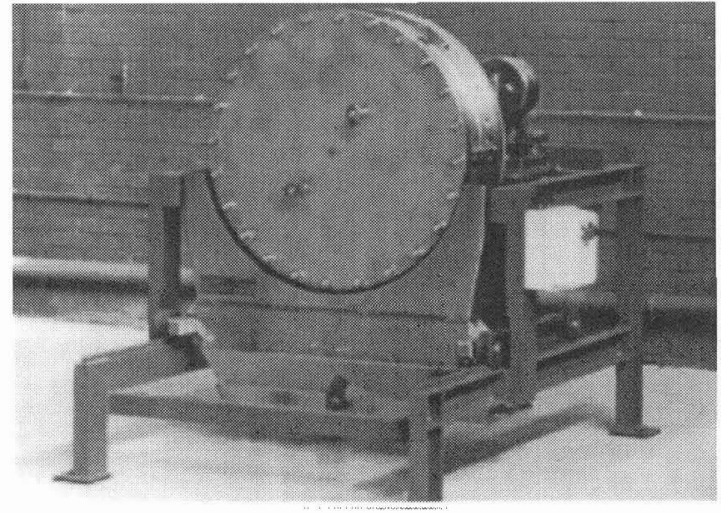

Figure 1: Broyeur à boulets utilisé pour la fabrication des alliages FeAl40 Grade 3.

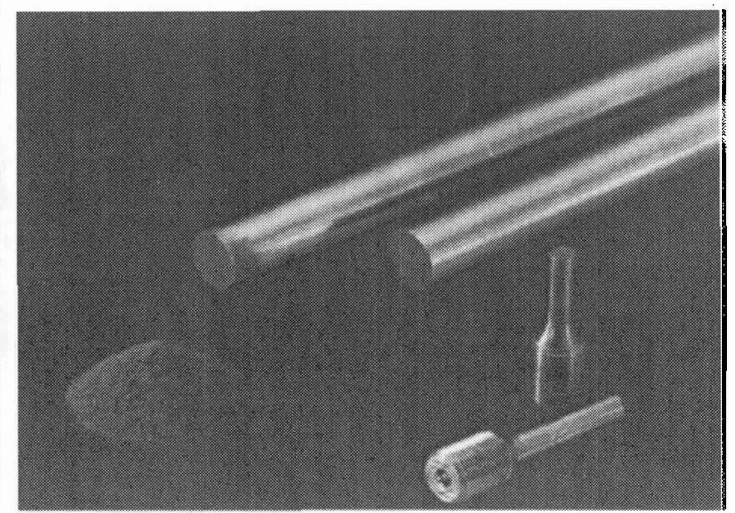

Figure 2: Poudre broyée et barres extrudées d'alliage FeAl40 Grade 3 . 


\section{MICROSTRUCTURE ET RECRISTALLISATION DES ALLIAGES FeAI40 Grade 3}

\subsection{Recristallisation et texture}

Les conditions de recristallisation entre 1000 et $1300^{\circ} \mathrm{C}$ ont été étudiées sur des échantillons de $10 \mathrm{~mm}$ de diamètre et $3 \mathrm{~mm}$ de hauteur bruts d'extrusion à l'aide de recuits isochrones d'une heure en four sous balayage d'argon [21].

L'alliage FeAl40 Grade 3 brut d'extrusion présente une structure très fine (fig. 3.a). La figure de pôle révèle une forte texture de fibres $<110>$ parallèle à l'axe d'extrusion (fig. 3.b). Aucune modification de la structure et de la texture n'est observée après un traitement thermique jusqu'à $1000^{\circ} \mathrm{C}$. Les échantillons traités à 1100 et $1200^{\circ} \mathrm{C}$ conservent une structure très fine, similaire à celle du matériau brut, à l'exception d'un renforcement de la texture de fibres. La recristallisation primaire du matériau observée dans ce cas permet d'obtenir une texture de fibres $<110>$ plus homogène. Les échantillons traités à $1250^{\circ} \mathrm{C}$ présentent une distribution bimodale de tailles de grains avec une majorité de grains fins et une faible proportion de gros grains dont la taille peut atteindre $100 \mu \mathrm{m}$ dans le sens transversal. La figure de pôle montre une intense texture $\langle 110\rangle$, mais aussi des pics $<111>$ correspondant à la présence des gros grains. Après un traitement thermique à $1300^{\circ} \mathrm{C}$, la structure est composée principalement de gros grains $(200 \mu \mathrm{m}$ de dimension transversale et plus de $500 \mu \mathrm{m}$ dans le sens d'extrusion). La texture $<110>$ a été remplacée par des renforcements suivant $<111>$ et $<211>$.

La texture $<110>$ observée dans le matériau brut d'extrusion explique la valeur de 205 Gpa du module d'Young mesuré en traction sur ces barres. Cette valeur est en effet inférieure à celle mesurée sur des barres extrudées à $1000^{\circ} \mathrm{C}$ d'alliages $\mathrm{FeAl}$ non renforcés par des dispersoïdes, et qui présentent une texture $<111>$ favorable à la rigidité $[22,23-24]$. Sur les alliages renforcés, la texture $<111>$ peut être retrouvée par traitement thermique mais au prix d'un grossissement du grain défavorable à la ductilité.

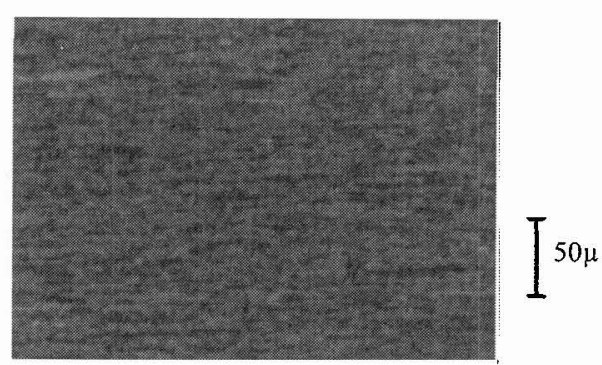

a)

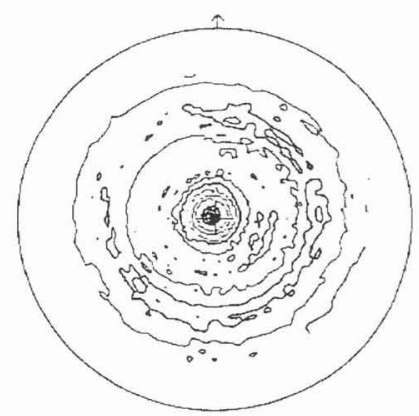

b)

Figure 3: a) Micrographie optique et b) figures de pôles 110 de l'alliage FeAl40 Grade 3 brut d'extrusion.

\subsection{Microstructure observée en Microscopie Electronique à Transmission (MET)}

Les alliages FeAl40 Grade 3 présentent une structure très fine également à l'échelle du MET [21]. Ces alliages ont une taille de grains de 1 à $2,5 \mu \mathrm{m}$ suivant l'axe d'extrusion et de 0,5 à $1 \mu \mathrm{m}$ dans le sens perpendiculaire (fig. 4).

Deux familles de particules renforçantes sont observés et ont été analysées par EDS. 


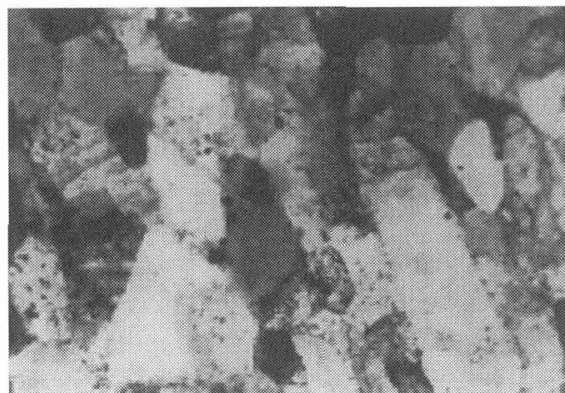

a)

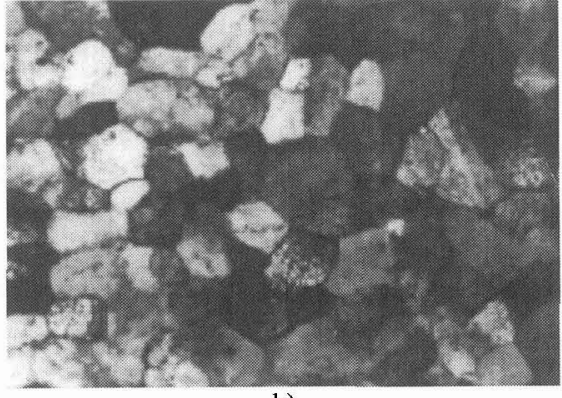

b)

Figure 4: Microstructure de l'alliage FeA140 Grade 3 brut d'extrusion observée en MET. a) Sens parallèle à l'axe d'extrusion. b) Sens perpendiculaire à l'axe d'extrusion.

En premier lieu, de grandes particules d'oxyde mixte d'yttrium (majoritaire) et d'aluminium, de morphologie plutôt sphérique et de taille comprise entre 50 et $200 \mathrm{~nm}$, sont présentes principalement aux joints de grains. Ces particules garantissent la tenue à la recristallisation et améliorent la tenue au fluage à haute température en réduisant les possibilités de glissement intergranulaire.

En second lieu, les dispersoïdes intragranulaires sont de taille comprise entre 20 et $30 \mathrm{~nm}$ et sont des oxydes d'yttrium, d'aluminium et de fer. Par le mécanisme d'Orowan, ces dispersoïdes sont responsables de la résistance à la rupture et au fluage de la matrice, et donc de l'alliage dans le domaine des températures basses et moyennes jusqu'à $700^{\circ} \mathrm{C}[25]$.

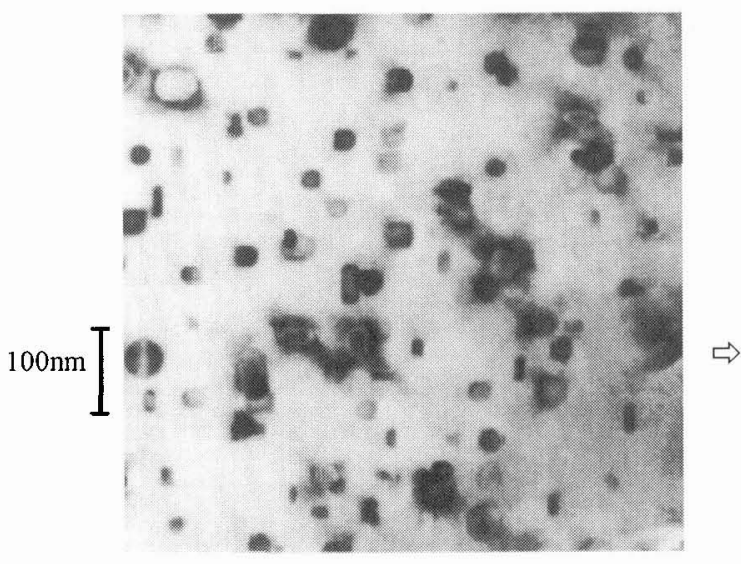

a)

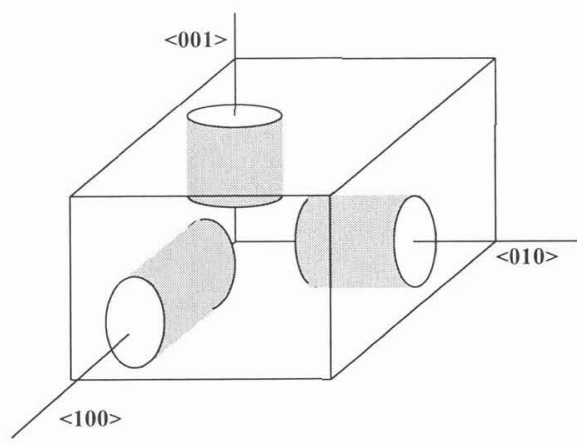

b)

Figure 5: a) Observation en MET en axe de zone 100 des dispersoïdes intragranulaires dans l'alliage FeAl40 Grade 3 brut d'extrusion. b) Relation d'orientation géométrique entre l'axe des dispersoïdes et les plans de la matrice.

Une vue en axe de zone 100 de la matrice (fig. 5.a) montre que ces dispersoïdes présentent des franges de moiré et une forme cylindrique (schématiquement reproduite en fig. 5.b) avec un grand axe parallèle aux plans $\{100\}$ de la matrice. Les figures de diffraction confirment l'existence d'une relation d'orientation, les plans $\{111\}$ des dispersoïdes étant parallèles aux plans $\{100\}$ de la matrice. Cette cohérence partielle est à mettre en relation avec l'écart relativement faible $(<5 \%)$ de paramètre de maille entre ces familles de plans de $\mathrm{Y}_{2} \mathrm{O}_{3}$ et $\mathrm{FeAl}$. 
Cette cohérence explique également qu'au cours d'un traitement thermique de $1200^{\circ} \mathrm{C}$ pendant une heure, ces dispersö̈des coalescent jusqu'à une taille de $50 \mathrm{~nm}$ et s'enrichissent en aluminium et fer, perdant ainsi une partie de leur caractère renforçant.

\section{PROPRIETES MECANIQUES DES ALLIAGES FeAI40 Grade 3}

Les propriétés mécaniques des alliages FeAl40 Grade 3 ont été déterminées dans les conditions expérimentales les moins favorables à la ductilité: sur éprouvettes usinées non polies, sous air non déshydraté et à une vitesse de traction faible. Les courbes de traction sont présentées en figure 6 et comparées à celle d'un alliage $\mathrm{Fe}-40 \% \mathrm{Al}-\mathrm{Zr}-\mathrm{B}$ non renforcé par des dispersoïdes et possédant la même composition que la matrice des alliages FeAl40 Grade 3. Les avantages en terme de résistance mécanique et de ductilité des alliages FeAl40 Grade 3 à grains fins et renforcés sont très nets.
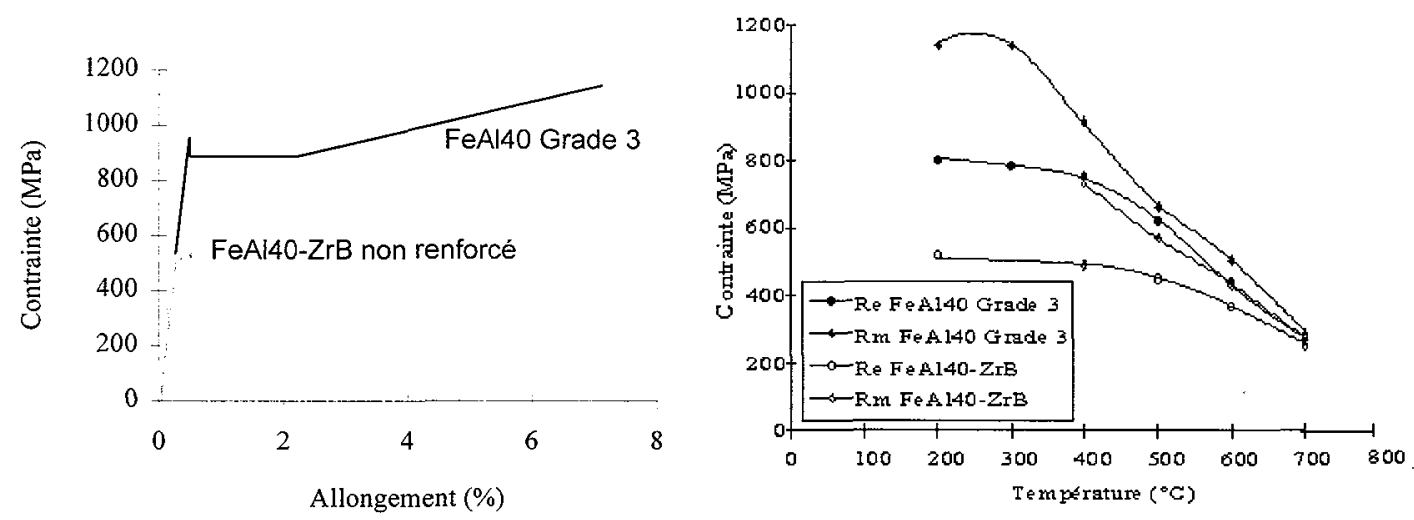

Figure 6: Courbes de traction de l'alliage FeAl40 Grade 3 comparée à la courbe de traction d'un alliage $\mathrm{Fe}-40 \% \mathrm{Al}-\mathrm{Zr}-\mathrm{B}$ non renforcé.

Figure 7: Limites élastiques $(\mathrm{Re})$ et à rupture $(\mathrm{Rm})$ des alliages FeA140 Grade 3-B et Fe-40\%Al-Zr-B non renforcé en fonction de la température.

La comparaison des limites élastiques et à rupture en fonction de la température (fig. 7) montre que l'effet du renforcement par dispersoïdes est sensible jusqu'à $700^{\circ} \mathrm{C}$. Au delà, la faible taille de grains de l'alliage renforcé réduit la résistance mécanique par glissement intergranulaire.

Les propriétés en fatigue des alliages FeAl40 Grade 3 présentent des niveaux comparables à celles d'un acier allié [26]. A $500^{\circ} \mathrm{C}$ à l'air, la rupture en fluage se produit sous $440 \mathrm{MPa}$ au bout de 100 heures. A $700^{\circ} \mathrm{C}$ et sous $55 \mathrm{MPa}$, le renforcement par dispersion d'oxyde réduit de 3 ordres de grandeur la vitesse de déformation en fluage.

\section{APPLICATIONS DES ALLIAGES FeAl40 Grade 3}

Les propriétés physiques et mécaniques à 20 et $500^{\circ} \mathrm{C}$ de l'alliage FeAl40 Grade 3 sont comparées à celles d'autres alliages structuraux industriels dans le tableau 1.

Par rapport aux autres alliages structuraux, les points forts des alliages FeAl40 Grade 3 sont:

- une faible densité et une résistance spécifique élevée comparée aux aciers et aux superalliages, 
- une rigidité spécifique élevée comparée aux alliages légers, aux aciers et aux alliages de nickel,

- une ductilité élevée comparées à celle des autres intermétalliques (TiAl, NiAl),

- une résistance mécanique élevée jusqu'à $700^{\circ} \mathrm{C}$ par rapport aux alliages d'aluminium et aux composites à matrice polymère,

- une résistance à la corrosion sèche élevée par rapport à la plupart des aciers et superalliages inoxydables.

\begin{tabular}{|c|c|c|c|c|c|c|}
\hline & $\begin{array}{c}\text { FeAl40 } \\
\text { Grade } 3 \\
\text { brut d'extr. }\end{array}$ & $\begin{array}{c}\text { Alliage d' } \\
\text { aluminium } \\
2024(\mathrm{~T} 6)\end{array}$ & $\begin{array}{c}\text { Alliage de } \\
\text { titane } \\
\text { TA6V }\end{array}$ & $\begin{array}{c}\text { Acier } \\
\text { inoxydable } \\
316 \mathrm{~L}\end{array}$ & $\begin{array}{c}\text { Alliage de } \\
\text { nickel } \\
625\end{array}$ & $\begin{array}{l}\text { Super- } \\
\text { alliage } \\
\text { IN100 }\end{array}$ \\
\hline Densité & 5.9 & 2.77 & 4.43 & 8 & 8.44 & 7.75 \\
\hline Temp. de fusion $\left({ }^{\circ} \mathrm{C}\right)$ & 1310 & 502 & 1600 & 1375 & 1290 & \\
\hline Coef. d'expan. thermique $\left(10^{-6} /{ }^{\circ} \mathrm{C}\right)$ & 25 & 22 & 9 & 15 & 12.8 & 13 \\
\hline Conductivité thermique $(\mathrm{W} / \mathrm{m} . \mathrm{K})$ & 12 & 151 & 7 & 16 & 10 & 17 \\
\hline$\underline{20^{\circ} \mathrm{C}}$ & & & & & & \\
\hline Limite élastique ( $M P a)$ & 894 & 393 & 860 & $170-310$ & 517 & 850 \\
\hline Limite à rupture $(M P a)$ & 1147 & 476 & 930 & $480-620$ & 930 & 1010 \\
\hline Allongement (\%) & 6.4 & 10 & 13 & $30-40$ & 43 & 9 \\
\hline Module d'Young (GPa) & 200 & 72 & 114 & $190-215$ & 207 & 215 \\
\hline $\begin{array}{l}\text { Rigidité spécifique } \sqrt{\mathrm{E} / \rho} \\
\left(M P a / g . \mathrm{cm}^{-3}\right)^{1 / 2}\end{array}$ & 184 & 161 & 156 & $154-164$ & 157 & 167 \\
\hline $\begin{array}{l}\text { Résistance spécifique } \mathrm{Re} / \rho \\
\left(\mathrm{MPa} / \mathrm{g} . \mathrm{cm}^{-3}\right)\end{array}$ & 188 & 142 & 191 & $21-39$ & 61 & 110 \\
\hline$\underline{500^{\circ} \mathrm{C}}$ & & & & & & \\
\hline Limite élastique (MPa) & 663 & & $500-550$ & 108 & 405 & 885 \\
\hline Limite à rupture $(\mathrm{MPa})$ & 704 & & $600-770$ & & 745 & 1090 \\
\hline Allongement (\%) & 32 & & 22 & & 50 & \\
\hline Module d'Young (GPa) & 110 & & 91 & 155 & & 190 \\
\hline $\begin{array}{l}\text { Rigidité spécifique } \sqrt{\mathrm{E} / \rho} \\
\left(\mathrm{MPa} / \mathrm{g}_{\mathrm{cm}} \mathrm{cm}^{-3}\right)^{1 / 2}\end{array}$ & 139 & & 143 & 140 & & 158 \\
\hline $\begin{array}{l}\text { Résistance spécifique } \mathrm{Re} / \rho \\
\left(\mathrm{MPa} / \mathrm{g} . \mathrm{cm}^{-3}\right)\end{array}$ & 112 & & $111-122$ & & & 114 \\
\hline
\end{tabular}

Tableau 1: Propriétés physiques et mécaniques de l'alliage FeAl40 Grade 3 brut d'extrusion comparées à celles d'autres alliages industriels.

Quoique l'ensemble des propriétés d'usage des alliages FeAl40 Grade 3 ne soit pas encore connu, il est possible de considérer ces matériaux comme des substituts possibles aux alliages légers, aux aciers ou aux superalliages pour des applications industrielles exploitant leurs propriétés particulières:

- La densité réduite de $25 \%$ par rapport aux aciers et alliages de nickel, à propriétés et moyens de mise en oeuvre comparables par ailleurs, permet d'envisager la réduction de poids de pièces structurales aéronautiques et spatiales: boulonneries, trains d'atterrissage, pièces de systèmes de freinage, ...

- La résistance spécifique élevée permet également d'envisager des applications en substitution d'alliages à haute résistance (aciers et superalliages) utilisés pour la fabrication de pièces critiques en mouvement de moteurs thermiques et de turbomachines, tels que les soupapes, les axes et arbres, les aubes de turbines. La réduction de masse de tels composants réduit généralement les problèmes d'inertie, de frottement et de vibration et entraine de ce fait la réduction de masse d'autres 
composants tels que les paliers, les ressorts, les systèmes d'attache et de refroidissement, par effet «boule de neige ».

- La rigidité spécifique constitue une propriété particulièrement intéressante de ces matériaux. Elle est en effet 10 à $20 \%$ plus élevée que celle des alliages structuraux utilisés actuellement (alliages légers, aciers et superalliages) pour la fabrication de pièces devant travailler dans des régimes vibratoires proches de limites de résonance, voire au delà, telles que certains arbres de puissance de turbines ou certaines buses ou canalisations d'injection de fluides.

- Les propriétés de résistance à la corrosion peuvent être utilisées dans la fabrication de résistors de fours ou de tubes d'échangeurs de chaleur.

\section{CONCLUSIONS}

- A partir d'un modèle du comportement des alliages fer-aluminium intégrant les connaissances existantes sur les mécanismes régissant leurs propriétés, et en particulier la fragilité à température ambiante, une nouvelle famille d'alliages présentant une ductilité et une résistance élevées de 20 à $600^{\circ} \mathrm{C}$ est développée. Ses propriétés sont basées sur une composition optimisée pour renforcer les joints de grains, une taille de grains faible pour augmenter la ductilité et, associée à une dispersion d'oxyde, maximiser la résistance mécanique. Ces alliages FeA140 Grade 3 sont obtenus par une voie de métallurgie des poudres incluant une étape de broyage.

- L'étude en MET et en DRX de ces alliages bruts d'extrusion et traités thermiquement révèle la finesse de la dispersion d'oxyde partiellement cohérente avec la matrice et l'existence d'une structure de grains très fine $(0,5 \mu \mathrm{m})$, stable jusqu'à $1100^{\circ} \mathrm{C}$, et présentant une texture de fibres $<110>$.

- Outre les applications en corrosion, les propriétés mécaniques connues permettent d'envisager des applications structurales jusqu'à $600^{\circ} \mathrm{C}$ en substitution d'alliages industriels (alliages légers, aciers, superalliages) en particulier pour la fabrication de pièces en mouvement. La rigidité spécifique très élevée ouvre la voie à des applications sur pièces soumises à des régimes vibratoires critiques.

- Les alliages de la famille FeA140 Grade 3 ont été sélectionnés comme matériaux de référence dans le cadre de l'Action Concertée Européenne sur les Intermétalliques Structuraux (CEASI). A ce titre, ils font l'objet de diverses caractérisations dans divers laboratoires universitaires et industriels européens, en particulier dans les domaines de la résistance à haute température, du fluage, de la fatigue et de l'assemblage.

\section{Remerciements}

Les auteurs Mme AURIC, du CEA/DSM/DRFMC et M. LEFORT, du CEA/DTA/CEREM/DEM pour les discussions fructueuses qu'ils leurs ont accordé, ainsi que MM. BIGAY et CHALAYE, du CEA/DTA/CEREM/CE2M/LETRAM, pour l'extrusion du matériau, Mme HOSTACHE et MM. SAN FILIPPO, GENTZBITTEL, HENNEQUIN, LABONNE et NOMBALAIS pour leur aide technique. 


\section{Références}

[1] Lefort A., Franzoni U., Tassa O., Dunstan D.G.R., Magnee A., Le Coze J., Cayla O., CEA/CENG, N.T. DEM n90/15, Progress Report, EEC Project n²038-87 (1990)

[2] Vedula K., Intermetallic Compounds: Vol.2, Practice. ed. by Westbrook J.H. and Fleischer R.L., (John Wiley \& Sons Ltd, 1994)

[3] Sainfort G., Mouturat P., Pépin P., Petit J., Cabane G., Salesse M., Mémoires Scientifiques de la Revue de Métallurgie, LX, $\mathrm{n}^{\circ} 2$ (1963) 125

[4] Crimp M.A., Vedula K., Mat. Sci. Eng. 78 (1986) 193

[5] Crimp M.A., Vedula K., High Temperature Ordered Intermetallic II, ed. by Stoloff N.S., Koch C.C., Liu C.T., Izumi O. (MRS Proc. Vol. 81, Pittsburgh, Pa., 1987) p.499

[6] Baker I., Gaidosh D.J., Mat. Sci. Eng. 96 (1987) 147

[7] Gaidosh D.J., Nathal M.V., Scripta Metall. Mater. 24 (1990) 1281

[8] Gaidosh D.J., Draper S.L., Nathal M.V., Met. Trans. A 20A (1989)1701

[9] Liu C.T., Lee E.H., McKamey C.G., Scripta Metall. Mater. 23 (1989) 875

[10] Liu C.T., George E.P., Scripta Metall. Mater. 24 (1990) 1285

[11] Mendiratta M.G., Kim H.M., Lipsitt H.A., Met. Trans. A 15A (1984) 395

[12] Feng C.R., Sadananda K., Scripta Metall. Mater. 24 (1990) 2107

[13] Crimp M.A., Vedula K., Phil. Mag. A 39 (1991) 559

[14] Baker I., Nagpal P., Liu F., Munroe P.R., Acta Metall. Mater. 39 (1991) 1637

[15] Nagpal P, Baker I., Scripta Metall. Mater. 25 (1991) 2577

[16] Webb G., Lefort A., Proc. ASM Materials Week, Pittsburgh, October 19-20 (1993)

[17] Lefort A., Webb G., Wolski K., Lecoze J., Thevenot F., Franzoni U., Tassa O., Proc. MATTEC-92, Grenoble, October 14-16, 1992, ed. by Niku-Lari A. (IITT-International, Paris, 1992) p.327

[18] Jacob V., Baccino R., Moret F., Suryanarayana C., Froes F.H., Mishurda J., Proc. 2nd International Conference on Mechanical Alloying for Structural Applications, Vancouver (1993) p.33

[19] Schröder J.H., Arzt E., Scripta Metall. Mater. 19 (1985) 1129

[20] Baccino R., San Filippo D., Moret F., Lefort A., Webb G., Proc. of Powder Metallurgy World Congress PM'94, Paris, June 6-9, 1994, Vol. II (Ed. de Physique, Paris, 1994) p.1239

[21] Martel, P., CEA/CENG, Rapport Interne (1995)

[22] Stout J.J., Crimp M.A., Mater. Sci. Eng. A152 (1992) pp.335-340

[23] Harmouche M.R., Wolfenden A., Mat. Sci. Eng. 84 (1986) pp 35-42

[24] Munroe P.R., Baker I., J. Mater. Sci. 24 (1989) pp.4246-4252

[25] Morris D.G., Gunther S., "Strength and ductility of Fe-40Al Alloy prepared by mechanical alloying", Acta Metall. Mater., to be published.

[26] De Mestral, F., Communication au Colloque CEASI (Concerted European Action on Structural Intermetallics) du 25 mars 1995, Milan 